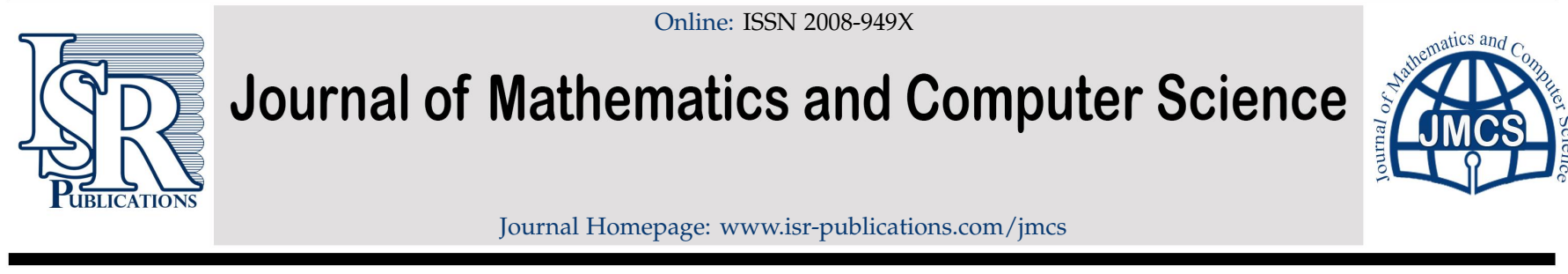

\title{
A new type of Zweier J-asymptotically lacunary statistically equivalent sequences
}

\author{
Vakeel A. Khan ${ }^{a, *}$, Abdullah A. H. Makharesh ${ }^{b}$, Masood Alam, Kamal M. A. S. Alshloola, Sameera A. A. \\ Abdullah ${ }^{\mathrm{a}}$ \\ ${ }^{a}$ Department of Mathematics, Aligarh Muslim University, Aligarh, 202002, India. \\ ${ }^{b}$ Department of Mathematics, Hadhramout University, Almahra, Yemen. \\ ${ }^{c}$ Department of Mathematics and IT Center for Preparatory Studies, Sultan Qaboos University, P. O. Box 162-PC, 123 Al Khoud Muscat, \\ Sultanate of Oman.
}

\begin{abstract}
In this article, by means of the Zweier matrix domain and modulus function we define and introduce some new definitions related to asymptotically equivalence for set sequences (Wijsman sense) in a metric space $(X, \rho)$ with respect to the ideal $\mathcal{J}$ of subset of natural numbers $\mathbb{N}$. In addition, we examine some results on these definitions.
\end{abstract}

Keywords: Zweier matrix, Wijsman asymptotically equivalence, modulus function, lacunary sequence.

2020 MSC: 40A35, 40C05.

(C)2020 All rights reserved.

\section{Introduction}

In 1980, Pobyvanets [28] introduced the concept of asymptotic equivalence which was further extended by Marouf in his work asymptotic equivalence and summability [24]. Patterson [26] and Patterson and Savas [27] defined the notion of asymptotic statistical equivalence and asymptotic lacunary statistical equivalence, respectively. Recently, the concept of asymptotic equivalence of sequence of numbers has been extended by several authors to asymptotic equivalence of sequences of sets. The one of these such extensions considered in this article is the concept of Wijsman asymptotically equivalence. In 2012 Ulusu and Nuray [33] defined asymptotically lacunary statistical equivalent set sequences and presented theorem about asymptotic equivalence (Wijsman sense). As we know that the notion of J-convergence is a generalization of the notion of statistical convergence which was introduced by Kostyrko at el. [22]. Since then this concept has become very important in field of classical analysis for more details about ideal and statistical convergence see, [10-12, 17-20]. Latterly, the notion of J-convergence has contributed to the development of a concept of asymptotically equivalent sequence of sets. One such development was the work provided by Kişi et al. [21] through which they introduced the notion of J-asymptotically

*Corresponding author

Email address: vakhanmaths@gmail .com (Vakeel A. Khan)

doi: $10.22436 /$ jmcs.021.04.07

Received: 2020-02-09 Revised: 2020-03-29 Accepted: 2020-04-14 
statistical equivalent and J-asymptotically lacunary statistical equivalent set sequences. For more details about asymptotically equivalence of sequence of sets in Wijsman sense see, [2, 6, 8, 9, 30-32].

The matrix domain has fundamental importance for this study. Sengonul [29] defined the sequence $y=\left(y_{i}\right)$ which is frequently used as the $z^{p}$-transform of the sequence $x=\left(x_{i}\right)$, i.e, $y_{i}=p x_{i}+(1-p) x_{i-1}$, where $x_{-1}=0,1<p<\infty$ and $z^{p}$ denoted the matrix $z^{p}=\left(z_{i k}\right)$ defined by

$$
z_{i k}= \begin{cases}p, & \text { if } i=k, \\ 1-p, & \text { if } i-1=k,(i, k \in \mathbb{N}) \\ 0, & \text { otherwise. }\end{cases}
$$

For more details on the use Zweier matrix in the theory of sequence space see, [3, 4, 13-16]. In this article, by means of the Zweier matrix domain, we introduce some new definitions as a generalization of asymptotically equivalence, that is, $z^{W}$-asymptotically equivalence, $z^{W}$-asymptotically statistically equivalence,

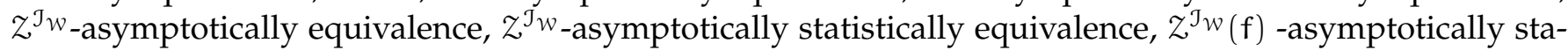

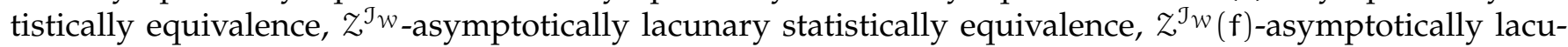
nary statistically equivalence, strongly $\mathcal{Z}^{\mathcal{J}_{\mathcal{W}}}$-asymptotically equivalence, $z^{\mathcal{J}_{\mathcal{W}}}$ (f)-asymptotically equivalence, strongly $Z^{J_{\mathcal{W}}}(\mathrm{f})$-asymptotically equivalence, $Z^{\mathcal{J}_{\mathcal{W}}}$-asymptotically lacunary equivalence and strongly $z^{\mathcal{J}} \mathcal{W}(f)$-asymptotically lacunary equivalence for set sequences in a metric space $(X, \rho)$. Furthermore, we study some inclusion theorems of these definitions.

\section{Definitions and preliminaries}

Throughout the article, let $(X, \rho)$ be a metric space. For any point $x \in X$ and non-empty subset $A$ of $X$, we define the distance from $x$ to $A$ by

$$
d(x, A)=\inf _{a \in A} \rho(x, a) .
$$

Definition 2.1 ([34]). Let $(X, \rho)$ be a metric space. For any non-empty closed subsets $A_{k}, B_{k} \subseteq X$, such that $d\left(x, A_{k}\right)>0$ and $d\left(x, B_{k}\right)>0$ for each $x \in X$, we say that the sequences $\left\{A_{k}\right\}$ and $\left\{B_{k}\right\}$ are said to be asymptotically equivalent (Wijsman sense) if for each $x \in X$,

$$
\lim _{k \rightarrow \infty} \frac{d\left(x, A_{k}\right)}{d\left(x, B_{k}\right)}=1
$$

and this is denoted by $A_{k} \sim B_{k}$.

Definition $2.2([34])$. Let $(X, \rho)$ be a metric space. For any non-empty closed subsets $A_{k}, B_{k} \subseteq X$, such that $d\left(x, A_{k}\right)>0$ and $d\left(x, B_{k}\right)>0$ for each $x \in X$, we say that the sequences $\left\{A_{k}\right\}$ and $\left\{B_{k}\right\}$ are said to be asymptotically statistically equivalent of multiple $L$ (Wijsman sense) if for every $\epsilon>0$ and for each $x \in X$,

$$
\lim _{n} \frac{1}{n}\left|\left\{k \leqslant n:\left|\frac{d\left(x, A_{k}\right)}{d\left(x, B_{k}\right)}-L\right| \geqslant \epsilon\right\}\right|=0
$$

and it is denoted by $A_{k} \stackrel{W S^{L}}{\sim} B_{k}$ and simply asymptotically statistical equivalent (Wijsman sense) if $L=1$.

Definition 2.3 ([22]). Let $X$ be a non-empty set then a family of sets $\mathcal{J} \subseteq 2^{X}$ is called an ideal in $X$ if and only if

(i) $\emptyset \in \mathcal{J}$;

(ii) for each $A, B \in \mathcal{J}$ we have $A \cup B \in \mathcal{J}$;

(iii) for each $A \in \mathcal{J}$ and each $B \subseteq A$ we have $B \in \mathcal{J}$. 
An ideal $\mathcal{J}$ is called non-trivial if $X \notin \mathcal{J}$ and a non-trivial ideal $\mathcal{J}$ is called an admissible in $X$ if and only if it contains all singletons, i.e., if $\mathcal{J} \supset\{\{x\}: x \in X\}$.

Definition 2.4 ([22]). A family of sets $\mathcal{F} \subseteq 2^{X}$ is a filter on $X$ if and only if

(i) $\emptyset \notin \mathcal{F}$;

(ii) for each $A, B \in \mathcal{F}$ we have $A \cap B \in \mathcal{F}$;

(iii) for each $A \in \mathcal{F}$ and $B \supseteq A$ we have $B \in \mathcal{F}$.

If $\mathcal{J}$ is a non-trivial ideal in $X$, then the family of sets $\mathcal{F}(\mathcal{J})=\{M \subset X: \exists A \in \mathcal{J}: M=X \backslash A\}$ is a filter on $X$ and it is called the filter associated with the ideal $\mathcal{J}$.

Definition $2.5([21])$. Let $(X, \rho)$ be a metric space and $\mathcal{J} \subset 2^{\mathbb{N}}$ be an admissible in $\mathbb{N}$. For any non-empty closed subsets $A_{k}, B_{k} \subseteq X$, such that $d\left(x, A_{k}\right)>0$ and $d\left(x, B_{k}\right)>0$ for each $x \in X$, we say that the sequences $\left\{A_{k}\right\}$ and $\left\{B_{k}\right\}$ are said to be Wijsman J-asymptotically equivalent of multiple $L$ if for every $\epsilon>0$ and for each $x \in X$,

$$
\left\{k \in \mathbb{N}:\left|\frac{d\left(x, A_{k}\right)}{d\left(x, B_{k}\right)}-L\right| \geqslant \epsilon\right\} \in \mathcal{J}
$$

and it is denoted by $A_{k} \stackrel{J_{W}}{\sim} B_{k}$.

Definition $2.6([21])$. Let $(X, \rho)$ be a metric space and $\mathcal{J} \subset 2^{\mathbb{N}}$ be a non-trivial in $\mathbb{N}$. For any non-empty closed subsets $A_{k}, B_{k} \subseteq X$, we say that the sequence $\left\{A_{k}\right\}$ and $\left\{B_{k}\right\}$ are said to be strongly asymptotically equivalent of multiple $L$ (Wijsman sense ) with respect to the ideal $\mathcal{J}$ provided that every $\epsilon>0$, for each $x \in X$,

$$
\left\{n \in \mathbb{N}: \frac{1}{n} \sum_{k=1}^{n}\left|\frac{d\left(x, A_{k}\right)}{d\left(x, B_{k}\right)}-L\right| \geqslant \epsilon\right\} \in \mathcal{J} ;
$$

denoted by $A_{k} \stackrel{\mathcal{I}_{W}}{\sim} B_{k}$ and simply strongly asymptotically equivalent with respect to the ideal $\mathcal{J}$, if $L=1$.

Definition $2.7([7])$. Let $(X, \rho)$ be a metric space and $\mathcal{J} \subset 2^{\mathbb{N}}$ be a non-trivial ideal. For any non-empty closed subsets $A_{k}, B_{k} \subseteq X$ such that $d\left(x, A_{k}\right)>0$ and $d\left(x, B_{k}\right)>0$ for each $x \in X$. Two sequences $\left\{A_{k}\right\}$ and $\left\{B_{k}\right\}$ are said to be asymptotically Wijsman J-statistically equivalent of multiple L provided that for every $\epsilon>0$ and for every $\delta>0$,

$$
\left\{n \in \mathbb{N}: \frac{1}{n}\left|\left\{k \leqslant n:\left|\frac{d\left(x, A_{k}\right)}{d\left(x, B_{k}\right)}-L\right| \geqslant \epsilon\right\}\right| \geqslant \delta\right\} \in \mathcal{J},
$$

denoted by $A_{k} \stackrel{W^{\mathcal{J}}(S)^{\mathrm{L}}}{\sim} B_{k}$ and simply asymptotically Wijsman J-statistically equivalent if $L=1$.

In [25] Nakano introduced the notion of a modulus function as follows. By a modulus function, we mean a function $f$ from $[0 ; \infty)$ to $[0 ; \infty)$ such that

(i) $f(x)=0$ if and only if $x=0$;

(ii) $f(x+y) \leqslant f(x)+f(y)$ for all $x \geqslant 0, y \geqslant 0$;

(iii) $f$ is increasing;

(iv) $f$ is continuous from the right at 0 .

It follows from that $f$ must be continuous on $[0,1)$. A modulus function may be bounded or unbounded. Basarir [1], Maddox [23], Pehlivan and many others used a modulus function $f$ to define some new sequence spaces.

Definition 2.8 ([5]). A Lacunary sequence is an increasing integer sequence $\theta=\left\{k_{r}\right\}$ such that $k_{0}=0$ and $h_{r}=k_{r}-k_{r-1} \rightarrow \infty$ as $r \rightarrow \infty$. The intervals determined by $\theta$ will be denoted by $J_{r}=\left(k_{r-1}, k_{r}\right]$. 
Definition 2.9 ([21]). Let $(X, \rho)$ be a metric space and $\mathcal{J} \subset 2^{\mathbb{N}}$ be a non-trivial in $\mathbb{N}$. For any non-empty closed subsets $A_{k}, B_{k} \subseteq X$, we say that the sequences $\left\{A_{k}\right\}$ and $\left\{B_{k}\right\}$ are said to be strongly f-asymptotically lacunary equivalent of multiple $\mathrm{L}$ (Wijsman sense ) with respect to the ideal J provided that for each $\epsilon>0$, and $x \in X$,

$$
\left\{r \in \mathbb{N}: \frac{1}{h_{r}} \sum_{k \in I_{r}} f\left(\left|\frac{d\left(x, A_{k}\right)}{d\left(x, B_{k}\right)}-L\right|\right) \geqslant \epsilon\right\} \in \mathcal{J} ;
$$

denoted by $A_{k} \stackrel{N_{\theta}^{f}(\mathcal{J})}{\sim} B_{k}$ and simply strongly f-asymptotically lacunary equivalent with respect to the ideal $\mathrm{J}$, if $\mathrm{L}=1$.

Definition 2.10 ([7]). Let $(X, \rho)$ be a metric space and $\mathcal{J} \subset 2^{\mathbb{N}}$ be a non-trivial ideal. For any non-empty closed subsets $A_{k}, B_{k} \subseteq X$ such that $d\left(x, A_{k}\right)>0$ and $d\left(x, B_{k}\right)>0$ for each $x \in X$. We say that the sequences $\left\{A_{k}\right\}$ and $\left\{B_{k}\right\}$ are said to be asymptotically Wijsman J-lacunary statistically equivalent (or $\mathcal{J}\left(S_{\theta}\right)$-equivalent) of multiple L provided that for every $\epsilon>0$, for every $\delta>0$ and for each $x \in X$,

$$
\left\{r \in \mathbb{N}: \frac{1}{h_{r}}\left|\left\{k \in I_{r}:\left|\frac{d\left(x, A_{k}\right)}{d\left(x, B_{k}\right)}-L\right| \geqslant \epsilon\right\}\right| \geqslant \delta\right\} \in \mathcal{J},
$$

denoted by $A_{k} \underset{\sim \mathcal{J}\left(S_{\theta}\right)^{L}}{\sim} B_{k}$ and simply asymptotically Wijsman $\mathcal{J}\left(S_{\theta}\right)$-equivalent if $L=1$.

\section{Main results}

Throughout the article, for the sake of convenience now we will denote by $z^{p}\left(A_{k}\right)=A_{k}^{\prime}, z^{p}\left(B_{k}\right)=B_{k}^{\prime}$. Let $(X, \rho)$ be a metric space. For non-empty closed subsets $A_{k}, B_{k} \subseteq X$, by means of the Zweier matrix domain we define $d\left(x, A_{k}^{\prime}, B_{k}^{\prime}\right)$ as follows:

$$
d\left(x, A_{k}^{\prime}, B_{k}^{\prime}\right)= \begin{cases}\frac{d\left(x, A_{k}^{\prime}\right)}{d\left(x, B_{k}^{\prime}\right)}, & \text { if } x \notin A_{k}^{\prime} \cup B_{k^{\prime}}^{\prime} \\ L, & \text { if } x \in A_{k}^{\prime} \cup B_{k^{\prime}}^{\prime}\end{cases}
$$

We now consider our main results. We begin with the following definitions.

Definition 3.1. Let $(X, \rho)$ be a metric space. For any non-empty closed subsets $A_{k}, B_{k} \subseteq X$, such that $d\left(x, A_{k}\right)>0$ and $d\left(x, B_{k}\right)>0$ for each $x \in X$, we say that the sequences $\left\{A_{k}\right\}$ and $\left\{B_{k}\right\}$ are said to be Zweier asymptotically equivalent (Wijsman sense), if for each $x \in X$,

$$
\lim _{k \rightarrow \infty} d\left(x, A_{k}^{\prime}, B_{k}^{\prime}\right)=L
$$

and it is denoted by $A_{k} \stackrel{z^{W}}{\sim} B_{k}$ and simply $z^{W}$-asymptotically equivalent, if $L=1$.

Definition 3.2. Let $(X, \rho)$ be a metric space. For any non-empty closed subsets $A_{k}, B_{k} \subseteq X$, such that $d\left(x, A_{k}\right)>0$ and $d\left(x, B_{k}\right)>0$ for each $x \in X$, we say that the sequences $\left\{A_{k}\right\}$ and $\left\{B_{k}\right\}$ are said to be Zweier asymptotically statistically equivalent of multiple L (Wijsman sense), if for every $\epsilon>0$ and for each $x \in X$,

$$
\lim _{n} \frac{1}{n}\left|\left\{k \leqslant n:\left|d\left(x, A_{k}^{\prime}, B_{k}^{\prime}\right)-L\right| \geqslant \epsilon\right\}\right|=0
$$

and it is denoted by $A_{k} \stackrel{z^{W}(S)}{\sim} B_{k}$ and simply $z^{W}$-asymptotically statistically equivalent, if $L=1$. 
Definition 3.3. Let $(X, \rho)$ be a metric space and $\mathcal{J} \subset 2^{\mathbb{N}}$ be an admissible ideal. For any non-empty closed subsets $A_{k}, B_{k} \subseteq X$, such that $d\left(x, A_{k}\right)>0$ and $d\left(x, B_{k}\right)>0$ for each $x \in X$, we say that the sequences $\left\{A_{k}\right\}$ and $\left\{B_{k}\right\}$ are said to be Zweier asymptotically equivalent of multiple $L$ (Wijsman sense) with respect to the ideal $\mathcal{J}$, if for every $\epsilon>0$ and for each $x \in X$,

$$
\left\{k \in \mathbb{N}:\left|d\left(x, A_{k}^{\prime}, B_{k}^{\prime}\right)-L\right| \geqslant \epsilon\right\} \in \mathcal{J}
$$

and it is denoted by $A_{k} \stackrel{z^{J} W}{\sim} B_{k}$ and simply $z^{J^{J}}$-asymptotically equivalent, if $L=1$.

Definition 3.4. Let $(X, \rho)$ be a metric space and $\mathcal{J} \subset 2^{\mathbb{N}}$ be an admissible ideal. For any non-empty closed subsets $A_{k}, B_{k} \subseteq X$ such that $d\left(x, A_{k}\right)>0$ and $d\left(x, B_{k}\right)>0$ for each $x \in X$, we say that the sequences $\left\{A_{k}\right\}$ and $\left\{B_{k}\right\}$ are said to be Zweier asymptotically statistically equivalent of multiple L (Wijsman sense) with respect to the ideal $\mathcal{J}$, if for every $\epsilon>0$ and for every $\delta>0$,

$$
\left\{n \in \mathbb{N}: \frac{1}{n}\left|\left\{k \leqslant n:\left|d\left(x, A_{k}^{\prime}, B_{k}^{\prime}\right)-L\right| \geqslant \epsilon\right\}\right| \geqslant \delta\right\} \in \mathcal{J}
$$

and it is denoted by $A_{k} \stackrel{Z^{J} W(S)}{\sim} B_{k}$ and simply $Z^{\mathcal{J} W}$-asymptotically statistically equivalent, if $L=1$.

Definition 3.5. Let $(X, \rho)$ be a metric space, $\mathcal{J} \subset 2^{\mathbb{N}}$ be an admissible ideal and $f$ be modulus function. For any non-empty closed subsets $A_{k}, B_{k} \subseteq X$ such that $d\left(x, A_{k}\right)>0$ and $d\left(x, B_{k}\right)>0$ for each $x \in X$, we say that the sequences $\left\{A_{k}\right\}$ and $\left\{B_{k}\right\}$ are said to be Zweier $f$-asymptotically statistically equivalent of multiple L (Wijsman sense) with respect to the ideal $\mathcal{J}$, if for every $\epsilon>0$ and for every $\delta>0$,

$$
\left\{n \in \mathbb{N}: \frac{1}{n}\left|\left\{k \leqslant n: f\left(\left|d\left(x, A_{k}^{\prime}, B_{k}^{\prime}\right)-L\right|\right) \geqslant \epsilon\right\}\right| \geqslant \delta\right\} \in \mathcal{J}
$$

and it is denoted by $A_{k} \stackrel{Z^{J} W\left(S^{f}\right)}{\sim} B_{k}$ and simply $Z^{J} w(f)$-asymptotically statistically equivalent, if $L=1$.

Definition 3.6. Let $(X, \rho)$ be a metric space, $\mathcal{J} \subset 2^{\mathbb{N}}$ be an admissible ideal and $\theta$ be a lacunary sequence. For any non-empty closed subsets $A_{k}, B_{k} \subseteq X$ such that $d\left(x, A_{k}\right)>0$ and $d\left(x, B_{k}\right)>0$ for each $x \in X$, we say that the sequences $\left\{A_{k}\right\}$ and $\left\{B_{k}\right\}$ are said to be Zweier asymptotically lacunary statistically equivalent of multiple L (Wijsman sense) with respect to the ideal $\mathcal{J}$, if for every $\epsilon>0$, for every $\delta>0$ and for each $x \in X$,

$$
\left\{r \in \mathbb{N}: \frac{1}{h_{r}}\left|\left\{k \in I_{r}:\left|d\left(x, A_{k}^{\prime}, B_{k}^{\prime}\right)-L\right| \geqslant \epsilon\right\}\right| \geqslant \delta\right\} \in \mathcal{J}
$$

and it is denoted by $A_{k} \stackrel{z^{J} W\left(S_{\theta}\right)}{\sim} B_{k}$ and simply $z^{\mathcal{J} W}$-asymptotically lacunary statistically equivalent, if $\mathrm{L}=1$.

Definition 3.7. Let $(X, \rho)$ be a metric space, $\mathcal{J} \subset 2^{\mathbb{N}}$ be an admissible ideal, $\theta$ be a lacunary sequence and $f$ be a modulus function. For any non-empty closed subsets $A_{k}, B_{k} \subseteq X$ such that $d\left(x, A_{k}\right)>0$ and $d\left(x, B_{k}\right)>0$ for each $x \in X$, we say that the sequences $\left\{A_{k}\right\}$ and $\left\{B_{k}\right\}$ are said to be Zweier $f$-asymptotically lacunary statistically equivalent of multiple $L$ (Wijsman sense) with respect to the ideal $\mathcal{J}$, if for every $\epsilon>0$, for every $\delta>0$ and for each $x \in X$,

$$
\left\{r \in \mathbb{N}: \frac{1}{h_{r}}\left|\left\{k \in I_{r}: f\left(\left|d\left(x, A_{k}^{\prime}, B_{k}^{\prime}\right)-L\right|\right) \geqslant \epsilon\right\}\right| \geqslant \delta\right\} \in \mathcal{J}
$$

and it is denoted by $A_{k} \stackrel{z^{J} W\left(S_{\theta}^{f}\right)}{\sim} B_{k}$ and simply $z^{\mathcal{J} W}(f)$-asymptotically lacunary statistically equivalent, if $\mathrm{L}=1$. 
Definition 3.8. Let $(X, \rho)$ be a metric space and $\mathcal{J} \subset 2^{\mathbb{N}}$ be an admissible ideal. For any non-empty closed subsets $A_{k}, B_{k} \subset X$, we say that the sequences $\left\{A_{k}\right\}$ and $\left\{B_{k}\right\}$ are said to be strongly Zweier asymptotically equivalent of multiple $L$ (Wijsman sense) with respect to the ideal $\mathcal{J}$, if for every $\epsilon>0$, for each $x \in X$,

$$
\left\{n \in \mathbb{N}: \frac{1}{n} \sum_{k=1}^{n} d\left(x, A_{k}^{\prime}, B^{\prime}{ }_{k}\right)-L \geqslant \epsilon\right\} \in \mathcal{J}
$$

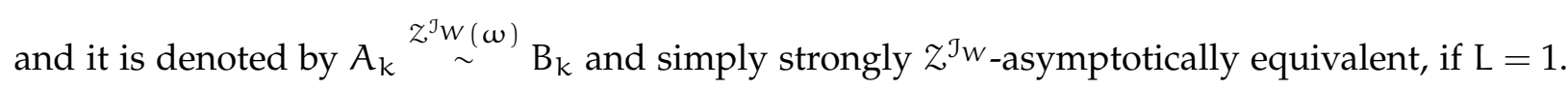

Definition 3.9. Let $(X, \rho)$ be a metric space, $\mathcal{J} \subset 2^{\mathbb{N}}$ be an admissible ideal and $f$ be a modulus function. For any non-empty closed subsets $A_{k}, B_{k} \subseteq X$, we say that the sequences $\left\{A_{k}\right\}$ and $\left\{B_{k}\right\}$ are said to be Zweier $f$-asymptotically equivalent of multiple $L$ (Wijsman sense) with respect to the ideal $\mathcal{J}$, if for every $\epsilon>0$, and for each $x \in X$,

$$
\left\{k \in \mathbb{N}: f\left(\left|d\left(x, A_{k}^{\prime}, B^{\prime}{ }_{k}\right)-L\right|\right) \geqslant \epsilon\right\} \in \mathcal{J}
$$

and it is denoted by $A_{k} \stackrel{Z^{J} W(f)}{\sim} B_{k}$ and simply $z^{J^{J}}(f)$-asymptotically equivalent, if $L=1$.

Definition 3.10. Let $(X, \rho)$ be a metric space, $\mathcal{J} \subset 2^{\mathbb{N}}$ be an admissible ideal and $f$ be a modulus function. For any non-empty closed subsets $A_{k}, B_{k} \subseteq X$, we say that the sequences $\left\{A_{k}\right\}$ and $\left\{B_{k}\right\}$ are said to be strongly Zweier $f$-asymptotically equivalent of multiple L (Wijsman sense) with respect to the ideal $\mathcal{J}$, if for every $\epsilon>0$, and for each $x \in X$,

$$
\left\{n \in \mathbb{N}: \frac{1}{n} \sum_{k=1}^{n} f\left(\left|d\left(x, A_{k}^{\prime}, B^{\prime}{ }_{k}\right)-L\right|\right) \geqslant \epsilon\right\} \in \mathcal{J}
$$

and it is denoted by $A_{k} \stackrel{z^{J} W\left(\omega_{f}\right)}{\sim} B_{k}$ and simply strongly $z^{J_{W}}(f)$-asymptotically equivalent, if $L=1$.

Definition 3.11. Let $(X, \rho)$ be a metric space, $\mathcal{J} \subset 2^{\mathbb{N}}$ be an admissible ideal and $\theta$ be a lacunary sequence. For any non-empty closed subsets $A_{k}, B_{k} \subseteq X$, we say that the sequences $\left\{A_{k}\right\}$ and $\left\{B_{k}\right\}$ are said to be strongly Zweier asymptotically lacunary equivalent of multiple L (Wijsman sense) with respect to the ideal $\mathcal{J}$, if for every $\epsilon>0$, and for each $x \in X$,

$$
\left\{r \in \mathbb{N}: \frac{1}{h_{r}} \sum_{k \in I_{r}}\left|d\left(x, A_{k}^{\prime}, B^{\prime}{ }_{k}\right)-L\right| \geqslant \epsilon\right\} \in \mathcal{J}
$$

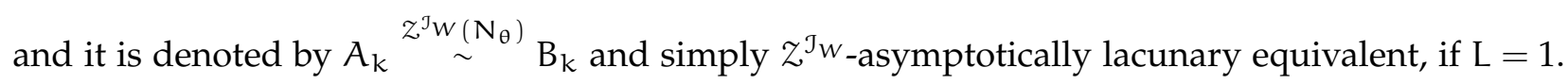

Definition 3.12. Let $(X, \rho)$ be a metric space, $\mathcal{J} \subset 2^{\mathbb{N}}$ be an admissible ideal in $\mathbb{N}$ and $f$ be a modulus function. For any non-empty closed subsets $A_{k}, B_{k} \subseteq X$, we say that the sequences $\left\{A_{k}\right\}$ and $\left\{B_{k}\right\}$ are said to be strongly Zweier f-asymptotically lacunary equivalent of multiple L (Wijsman sense) with respect to the ideal $\mathcal{J}$, if for every $\epsilon>0$, and for each $x \in X$,

$$
\left\{r \in \mathbb{N}: \frac{1}{h_{r}} \sum_{k \in I_{r}} f\left(\left|d\left(x ; A_{k}^{\prime}, B^{\prime}{ }_{k}\right)-L\right|\right) \geqslant \epsilon\right\} \in \mathcal{J}
$$

and it is denoted by $A_{k} \stackrel{z^{j} W\left(N_{\theta}^{f}\right)}{\sim} B_{k}$ and simply strongly $z^{J} W(f)$-asymptotically lacunary equivalent, if $\mathrm{L}=1$. 
Lemma 3.13. Let $\mathrm{f}$ be a modulus function and let $0<\delta<1$. Then for $\mathrm{y} \neq 0$ and each $\left(\frac{x}{y}\right)>\delta$, we have $f\left(\frac{x}{y}\right) \leqslant\left(\frac{2 f(1)}{\delta}\right)\left(\frac{x}{y}\right)$.

Theorem 3.14. let $\mathcal{J} \subset 2^{\mathbb{N}}$ be a non-trivial in $\mathbb{N}$ and $\mathrm{f}$ be a modulus function. Then,

(i) if $\mathrm{A}_{\mathrm{k}} \stackrel{z^{\top} w(\omega)}{\sim} \mathrm{B}_{\mathrm{k}}$, then $\mathrm{A}_{\mathrm{k}} \stackrel{z^{J} \mathrm{~W}\left(\omega_{\mathrm{f}}\right)}{\sim} \mathrm{B}_{\mathrm{k}}$;

(ii) $\lim _{\mathrm{t} \rightarrow \infty} \frac{\mathrm{f}(\mathrm{t})}{\mathrm{t}}=\alpha>0$, then $A_{k} \stackrel{z^{J} w(\omega)}{\sim} \mathrm{B}_{k} \Leftrightarrow A_{k} \stackrel{z^{j} w\left(\omega_{f}\right)}{\sim} B_{k}$.

Proof.

(i) Let $A_{k} \stackrel{Z^{J} w(\omega)}{\sim} B_{k}$, then we can write $\varepsilon>$ o be given. Choose $0<\delta<1$ such that $f(x)<\varepsilon$ for $0 \leqslant t \leqslant \delta$. Then we can write

$$
\frac{1}{n} \sum_{k=1}^{n} f\left(\left|d\left(x, A_{k}^{\prime} ; B_{k}^{\prime}\right)-L\right|\right)=\frac{1}{n} \sum_{\substack{k=1 \\\left|d\left(x, A_{k}^{\prime}, B_{k}^{\prime}\right)-L\right| \leqslant \delta}}^{n} f\left(\left|d\left(x, A_{k}^{\prime}, B_{k}^{\prime}\right)-L\right|\right)+\frac{1}{n} \sum_{\substack{k=1 \\\left|d\left(x, A_{k}^{\prime}, B_{k}^{\prime}\right)-L\right|>\delta}}^{n} f\left(\left|d\left(x, A_{k}^{\prime}, B_{k}^{\prime}\right)-L\right|\right) .
$$

Moreover, using the conditions of the modulus function $f$

$$
\frac{1}{n} \sum_{k=1}^{n} f\left(\left|d\left(x, A_{k}^{\prime}, B_{k}^{\prime}\right)-L\right|\right)<\epsilon+\left(\frac{2 f(1)}{\delta}\right) \frac{1}{n} \sum_{k=1}^{n}\left|d\left(x, A_{k}^{\prime}, B_{k}^{\prime}\right)-L\right| .
$$

Thus, for any $\gamma>0$,

$$
\left\{n \in \mathbb{N}: \frac{1}{n} \sum_{k=1}^{n} f\left(\left|d\left(x, A_{k}^{\prime}, B_{k}^{\prime}\right)-L\right|\right) \geqslant \gamma\right\} \subseteq\left\{n \in \mathbb{N}: \frac{1}{n} \sum_{k=1}^{n}\left|d\left(x, A_{k}^{\prime}, B_{k}^{\prime}\right)-L\right| \geqslant \frac{(\gamma-\epsilon) \delta}{2 f(1)}\right\} .
$$

Since $A_{k} \stackrel{z^{J} w(\omega)}{\sim} B_{k}$, it follows the later set, and hence, the first set in above expression belongs to J. This proves that $A_{k} \stackrel{z^{J} w\left(\omega_{f}\right)}{\sim} B_{k}$.

(ii) $\lim _{t \rightarrow \infty} \frac{f(t)}{t}=\alpha>0$, then we have $f(t) \geqslant \alpha t$ for all $t \geqslant 0$. Suppose that $A_{k} \stackrel{z^{\jmath} W\left(\omega_{f}\right)}{\sim} B_{k}$. Since

$$
\frac{1}{n} \sum_{k=1}^{n} f\left(\left|d\left(x, A_{k}^{\prime}, B_{k}^{\prime}\right)-L\right|\right) \geqslant \frac{1}{n} \sum_{k=1}^{n} \alpha\left(\left|d\left(x, A_{k}^{\prime}, B_{k}^{\prime}\right)-L\right|\right)=\alpha\left(\frac{1}{n} \sum_{k=1}^{n}\left|d\left(x, A_{k}^{\prime}, B_{k}^{\prime}\right)-L\right|\right),
$$

it follows that for each $\varepsilon>0$, we have

$$
\left\{n \in \mathbb{N}: \frac{1}{n} \sum_{k=1}^{n}\left|d\left(x, A_{k}^{\prime}, B_{k}^{\prime}\right)-L\right| \geqslant \varepsilon\right\} \subseteq\left\{n \in \mathbb{N}: \frac{1}{n} \sum_{k=1}^{n} f\left(\left|d\left(x, A_{k}^{\prime}, B_{k}^{\prime}\right)-L\right|\right) \geqslant \alpha \varepsilon\right\} .
$$

Since $A_{k} \stackrel{z^{J} W\left(\omega_{f}\right)}{\sim} B_{k}$, it follows that the later set belongs to $\mathcal{J}$, and therefore, the theorem is proved.

Theorem 3.15. Let $(X, \rho)$ be a metric space and $\left\{A_{k}\right\},\left\{B_{k}\right\}$ be two non-empty closed subset of $X(k \in \mathbb{N})$. Then

(i) $A_{k} \stackrel{Z^{J} w\left(N_{\theta}\right)}{\sim} B_{k} \Rightarrow A_{k} \stackrel{Z^{j} w\left(S_{\theta}\right)}{\sim} B_{k}$;

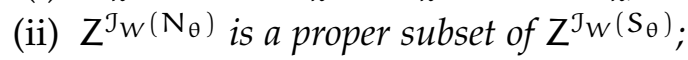

(iii) let $A_{k}, B_{k} \in L_{\infty}$ and $A_{k} \stackrel{Z^{J} W\left(S_{\theta}\right)}{\sim} B_{k}$;

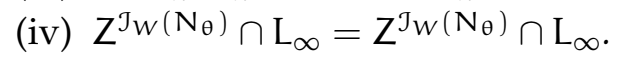


Proof.

(i) Let $\epsilon>0$ and $A_{k} \stackrel{Z^{J} W\left(N_{\theta}\right)}{\sim} B_{k}$. Then we can write

$$
\sum_{k \in J_{r}}\left|d\left(x, A_{k}^{\prime}, B_{k}^{\prime}\right)-L\right| \geqslant \sum_{\substack{k \in J_{r} \\\left|d\left(x, A_{k}^{\prime}, B_{k}^{\prime}\right)-L\right| \geqslant \epsilon}}\left|d\left(x, A_{k}^{\prime}, B_{k}^{\prime}\right)-L\right| \geqslant \epsilon\left|\left\{k \in J_{r}:\left|d\left(x, A_{k}^{\prime}, B_{k}^{\prime}\right)-L\right| \geqslant \epsilon\right\}\right| .
$$

If follows that

$$
\frac{1}{\epsilon \dot{\mathrm{h}}_{\mathrm{r}}} \sum_{\mathrm{k} \in \mathrm{J}_{\mathrm{r}}}\left|\mathrm{d}\left(x, \mathrm{~A}_{\mathrm{k}}^{\prime}, \mathrm{B}_{\mathrm{k}}^{\prime}\right)-\mathrm{L}\right| \geqslant \frac{1}{\mathrm{~h}_{\mathrm{r}}}\left|\left\{k \in \mathrm{J}_{\mathrm{r}}:\left|\mathrm{d}\left(x, \mathrm{~A}_{\mathrm{k}}^{\prime}, \mathrm{B}_{\mathrm{k}}^{\prime}\right)-\mathrm{L}\right| \geqslant \epsilon\right\}\right| .
$$

Thus for any $\delta>0$, we have

$$
\frac{1}{h_{r}}\left|\left\{k \in J_{r}:\left|d\left(x, A_{k}^{\prime}, B_{k}^{\prime}\right)-L\right| \geqslant \epsilon\right\}\right| \geqslant \delta
$$

Which implies that

$$
\frac{1}{h_{r}} \sum_{k \in J_{r}}\left|d\left(x, A_{k}^{\prime}, B_{k}^{\prime}\right)-L\right| \geqslant \epsilon \delta
$$

Therefore, we obtain

$$
\left\{r \in \mathbb{N}: \frac{1}{h_{r}}\left|\left\{k \in J_{r}:\left|d\left(x, A_{k}^{\prime}, B_{k}^{\prime}\right)-L\right| \geqslant \epsilon\right\}\right| \geqslant \delta\right\} \subset\left\{k \in \mathbb{N}: \frac{1}{h_{r}} \sum_{k \in J_{r}}\left|d\left(x, A_{k}^{\prime}, B_{k}^{\prime}\right)-L\right| \geqslant \epsilon \delta\right\} .
$$

Since $A_{k} \stackrel{Z^{J} W\left(N_{\theta}\right)}{\sim} B_{k}$, so that

$$
\left\{r \in \mathbb{N}: \frac{1}{h_{r}} \sum_{k \in J_{r}}\left|d\left(x, A_{k}^{\prime}, B_{k}^{\prime}\right)-L\right| \geqslant \epsilon \delta\right\} \in \mathcal{J} .
$$

Which implies that

$$
\left\{r \in \mathbb{N}: \frac{1}{h_{r}}:\left|\left\{k \in J_{r}:\left|d\left(x, A_{k}^{\prime}, B_{k}^{\prime}\right)-L\right| \geqslant \epsilon\right\}\right| \geqslant \delta\right\} \in \mathcal{J} .
$$

This shows that $A_{k} \stackrel{z^{j} w\left(S_{\theta}\right)}{\sim} B_{k}$.

(ii) Suppose that $A_{k} \stackrel{Z^{\jmath} W\left(N_{\theta}\right)}{\sim} B_{k} \subset A_{k} \stackrel{Z^{J} w\left(S_{\theta}\right)}{\sim} B_{k}$. Let $\left\{A_{k}\right\}$ and $\left\{B_{k}\right\}$ be two sequences of sets defined as follows:

$$
A_{k}=\left\{\begin{array}{l}
\{k\}, \quad \text { if } k_{r-1}<k<k_{r-1}+\left[\sqrt{h_{r}}\right], r=1,2,3, \ldots, \\
\{0\}, \quad \text { otherwise, }
\end{array}\right.
$$

and $B_{k}=1$ for all $k \in \mathbb{N}$. It is clear that $\left(A_{k}\right) \notin L_{\infty}$ and for $\epsilon>0$ and for each $x \in X$,

$$
\frac{1}{h_{r}}\left|\left\{k \in J_{r}:\left|d\left(x, A_{k}^{\prime}, B_{k}^{\prime}\right)-1\right| \geqslant \epsilon\right\}\right| \leqslant \frac{\left[\sqrt{h_{r}}\right]}{h_{r}} \text { and } \frac{\left[\sqrt{h_{r}}\right]}{h_{r}} \rightarrow 0 \text { as } r \rightarrow \infty .
$$

This implies that

$$
\left\{r \in \mathbb{N}: \frac{1}{h_{r}}\left|\left\{k \in J_{r}:\left|d\left(x, A_{k}^{\prime}, B_{k}^{\prime}\right)-1\right| \geqslant \epsilon\right\}\right| \geqslant \delta\right\} \subseteq\left\{r \in \mathbb{N}: \frac{\left[\sqrt{h_{r}}\right]}{h_{r}} \geqslant \delta\right\} .
$$


By virtue of last part of (3.15), the set on the right side is a finite set and so it belongs to J. Consequently, we have

$$
\left\{r \in \mathbb{N}: \frac{1}{h_{r}}\left|\left\{k \in J_{r}:\left|d\left(x, A_{k}^{\prime}, B_{k}^{\prime}\right)-1\right| \geqslant \epsilon\right\}\right| \geqslant \delta\right\} \in \mathcal{J} .
$$

Therefore $A_{k} \stackrel{z^{J} w\left(S_{\theta}\right)}{\sim} B_{k}$. On the other hand we shall show that $A_{k} \stackrel{z^{J} w\left(N_{\theta}\right)}{\sim} B_{k}$ is not satisfied. Suppose that $A_{k} \stackrel{z^{\jmath} W\left(N_{\theta}\right)}{\sim} B_{k}$. Then for every $\delta>0$, we have

$$
\left\{r \in \mathbb{N}: \frac{1}{h_{r}} \sum_{k \in J_{r}}\left|d\left(x, A_{k}^{\prime}, B_{k}^{\prime}\right)-1\right| \geqslant \delta\right\} \in \mathcal{J} .
$$

Now,

$$
\lim _{r} \frac{1}{h_{r}} \sum_{k \in J_{r}}\left|d\left(x, A_{k}^{\prime}, B_{k}^{\prime}\right)-1\right|=\frac{1}{h_{r}}\left(\frac{\left[\sqrt{h_{r}}\right]\left(\left[\sqrt{h_{r}}\right]-1\right)}{2}\right) \rightarrow \frac{1}{2} \text { as } r \rightarrow \infty .
$$

It follow that for the particular choice $\delta=\frac{1}{4}$,

$$
\left\{r \in \mathbb{N}: \frac{1}{h_{r}} \sum_{k \in J_{r}}\left|d\left(x, A_{k}^{\prime}, B_{k}^{\prime}\right)-1\right| \geqslant \frac{1}{4}\right\}=\left\{r \in \mathbb{N}:\left(\frac{\left[\sqrt{h_{r}}\right]\left(\left[\sqrt{h_{r}}\right]-1\right)}{h_{r}}\right) \geqslant \frac{1}{2}\right\}=\{m, m+1, m+2, \ldots\}
$$

for some $m \in \mathbb{N}$ which belong to $\mathcal{F}(\mathcal{J})$ as $\mathcal{J}$ is an admissible. This contradicts (3.1) for the choice $\delta=\frac{1}{4}$.

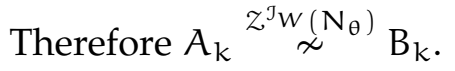

(iii) Suppose that $A_{k} \stackrel{z^{\jmath} w\left(S_{\theta}\right)}{\sim} B_{k}$ and $A_{k}, B_{k} \in L_{\infty}$. We assume that $\left|d\left(x, A^{\prime}, B_{k}^{\prime}\right)-L\right| \leqslant M$ for each $x \in X$ and for all $k \in \mathbb{N}$. Given $\epsilon>0$, we get

$$
\begin{aligned}
\frac{1}{h_{r}} \sum_{k \in J_{r}}\left|d\left(x, A_{k}^{\prime}, B_{k}^{\prime}\right)-L\right| & =\frac{1}{h_{r}} \sum_{\substack{k \in J_{r} \\
\left|d\left(x, A_{k^{\prime}}^{\prime} B_{k}^{\prime}\right)-L\right| \geqslant \epsilon}}+\frac{1}{h_{r}} \sum_{\substack{k \in J_{r} \\
\left|d\left(x, A_{k}^{\prime}, B_{k}^{\prime}\right)-L\right|<\epsilon}}\left|d\left(x, A_{k}^{\prime}, B_{k}^{\prime}\right)-L\right| \\
& \leqslant \frac{M}{h_{r}}\left|\left\{k \in J_{r}:\left|d\left(x, A_{k}^{\prime}, B_{k}^{\prime}\right)-L\right| \geqslant \epsilon\right\}\right|+\epsilon .
\end{aligned}
$$

If we put

$$
A(\epsilon)=\left\{r \in \mathbb{N}: \frac{1}{h_{r}} \sum_{k \in J_{r}}\left|d\left(x, A_{k}^{\prime}, B_{k}^{\prime}\right)-L\right| \geqslant \epsilon\right\}
$$

and

$$
B(\epsilon)=\left\{r \in \mathbb{N}: \frac{1}{h_{r}}\left|\left\{k \in J_{r}:\left|d\left(x, A_{k}^{\prime}, B_{k}^{\prime}\right)-L\right| \geqslant \epsilon\right\}\right| \geqslant \frac{\epsilon}{M}\right\},
$$

then we have $A(\epsilon) \subset B(\epsilon)$ and so $A(\epsilon) \in \mathcal{J}$. This shows that $A_{k} \stackrel{Z^{J} W\left(N_{\theta}\right)}{\sim} B_{k}$.

(iv) It follows from (i), (ii), and (iii).

Theorem 3.16. Suppose that for given $\delta>0$ and every $\epsilon>0$

$$
\left\{n \in \mathbb{N}: \frac{1}{n}\left|\left\{0 \leqslant k \leqslant n-1:\left|d\left(x, A_{k}^{\prime}, B_{k}^{\prime}\right)-L\right| \geqslant \epsilon\right\}\right|<\delta\right\} \in \mathcal{F}(\mathcal{J}) .
$$

Then $A_{k} \stackrel{Z^{J} W(S)}{\sim} B_{k}$. 
Proof. Let $\delta>0$ and every $\epsilon>0$ be given. For $\epsilon>0$, choose $n_{1}$ such that

$$
\frac{1}{n}\left|\left\{0 \leqslant k \leqslant n-1:\left|d\left(x, A_{k}^{\prime}, B_{k}^{\prime}\right)-L\right| \geqslant \epsilon\right\}\right|<\frac{\delta}{2} \text {, for all } n \geqslant n_{1} .
$$

It sufficient to show that there exist $n_{2}$ such that for $n \geqslant n_{2}$

$$
\frac{1}{n}\left|\left\{0 \leqslant k \leqslant n-1:\left|d\left(x, A_{k}^{\prime}, B_{k}^{\prime}\right)-L\right| \geqslant \epsilon\right\}\right|<\frac{\delta}{2} .
$$

Let $n_{0}=\max \left\{n_{1}, n_{2}\right\}$. The relation (3.2) will be true for $n>n_{0}$. If $m_{0}$ chosen fixed, then we get

$$
\left|\left\{0 \leqslant k \leqslant m_{0}-1:\left|d\left(x, A_{k}^{\prime}, B_{k}^{\prime}\right)-L\right| \geqslant \epsilon\right\}\right|=M .
$$

Now, for $n>m_{0}$, we have

$$
\begin{aligned}
\frac{1}{n}\left|\left\{0 \leqslant k \leqslant n-1:\left|d\left(x, A_{k}^{\prime}, B_{k}^{\prime}\right)-L\right| \geqslant \epsilon\right\}\right| & \leqslant \frac{1}{n}\left|\left\{0 \leqslant k \leqslant m_{0}-1:\left|d\left(x, A_{k}^{\prime}, B_{k}^{\prime}\right)-L\right| \geqslant \epsilon\right\}\right| \\
& +\frac{1}{n}\left|\left\{m_{0} \leqslant k \leqslant n-1:\left|d\left(x, A_{k}^{\prime}, B_{k}^{\prime}\right)-L\right| \geqslant \epsilon\right\}\right| \\
& \leqslant \frac{M}{n}+\frac{1}{n} \leqslant\left|\left\{m_{0} \leqslant k \leqslant n-1:\left|d\left(x, A_{k}^{\prime}, B_{k}^{\prime}\right)-L\right| \geqslant \epsilon\right\}\right| \\
& \leqslant \frac{M}{n}+\frac{\delta}{2} .
\end{aligned}
$$

Thus for sufficiently large $\mathrm{n}$

$$
\frac{1}{n} \leqslant\left|\left\{m_{0} \leqslant k \leqslant n-1:\left|d\left(x, A_{k}^{\prime}, B_{k}^{\prime}\right)-L\right| \geqslant \epsilon\right\}\right| \leqslant \frac{M}{n}+\frac{\delta}{2}<\delta .
$$

This establishes the result.

Theorem 3.17. Let $\mathcal{J}=\mathcal{J}_{\text {fin }}=\{A \subset \mathbb{N}: A$ is a finite set $\}$ be a non-trivial ideal. Let $(X, \rho)$ be a metric space and $\left\{A_{k}\right\},\left\{B_{k}\right\}$ be two non-empty closed subset of $X(k \in \mathbb{N})$. Let $\theta=\left\{k_{r}\right\}$ be a lacunary sequence with lim sup $\mathrm{q}_{r}<$ $\infty$. Then $A_{k} \stackrel{z^{J} W\left(S_{\theta}\right)}{\sim} B_{k} \Rightarrow A_{k} \stackrel{Z^{J} W(S)}{\sim} B_{k}$.

Proof. Omitted.

Theorem 3.18. Let $\mathcal{J} \subset 2^{\mathbb{N}}$ be a non-trivial in $\mathbb{N}$ and $\mathrm{f}$ be a modulus function. Then,

(i) if $A_{k} \stackrel{z^{J} W\left(S_{\theta}\right)}{\sim} B_{k}$, then $A_{k} \stackrel{z^{J} W(S)}{\sim} B_{k}$;

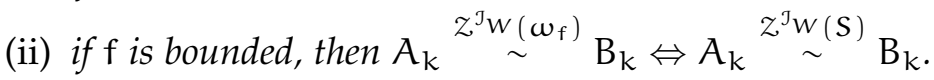

Proof.

(i) Suppose $A_{k} \stackrel{Z^{\jmath} W\left(\omega_{f}\right)}{\sim} B_{k}$, and $\varepsilon>0$ be given. Then we can write

$$
\frac{1}{n} \sum_{k=1}^{n} f\left(\left|d\left(x, A_{k}^{\prime}, B_{k}^{\prime}\right)-L\right|\right) \geqslant \frac{1}{n} \sum_{\substack{k=1 \\\left|d\left(x, A_{k}^{\prime}, B_{k}^{\prime}\right)-L\right| \geqslant \varepsilon}}^{n} f\left(\left|d\left(x, A_{k}^{\prime}, B_{k}^{\prime}\right)-L\right|\right) \geqslant \frac{f(\varepsilon)}{n}\left|\left\{k \leqslant n:\left|d\left(x, A_{k}^{\prime}, B_{k}^{\prime}\right)-L\right| \geqslant \varepsilon\right\}\right| .
$$

Therefore, for any $\gamma>0$, we have

$$
\left\{n \in \mathbb{N}: \frac{1}{n}\left\{|k \leqslant n:| d\left(x, A_{k}^{\prime}, B_{k}^{\prime}\right)-L|\geqslant \varepsilon|\right\} \geqslant \frac{\gamma}{f(\varepsilon)}\right\} \subseteq\left\{n \in \mathbb{N}: \frac{1}{n} \sum_{k=1}^{n} f\left(\left|d\left(x, A_{k}^{\prime}, B_{k}^{\prime}\right)-L\right|\right) \geqslant \gamma\right\} .
$$

Since $A_{k} \stackrel{Z^{J} W\left(\omega_{f}\right)}{\sim} B_{k}$, it follows that the later set belongs to J, and therefore $A_{k} \stackrel{Z^{J} W(S)}{\sim} B_{k}$. 
(ii) Assume that $f$ is bounded and $A_{k} \stackrel{Z^{J} W(S)}{\sim} B_{k}$. Since $f$ is bounded there exists a real number $M$ such that $\sup f(t) \leqslant M$. And for $\varepsilon>0$, we write

$$
\begin{aligned}
\frac{1}{n} \sum_{k=1}^{n} f\left(\left|d\left(x, A_{k}^{\prime}, B_{k}^{\prime}\right)-L\right|\right) & =\frac{1}{n}\left[\sum_{\substack{k=1 \\
\left|d\left(x, A_{k}^{\prime}, B_{k}^{\prime}\right)-L\right| \geqslant \varepsilon}}^{n} f\left(\left|d\left(x, A_{k}^{\prime}, B_{k}^{\prime}\right)-L\right|\right)+\sum_{\substack{k=1 \\
\left|d\left(x ; A_{k}^{\prime} ; B_{k}^{\prime}\right)-L\right|<\varepsilon}}^{n} f\left(\left|d\left(x, A_{k}^{\prime}, B_{k}^{\prime}\right)-L\right|\right)\right] \\
& \leqslant \frac{M}{n}\left|\left\{k \leqslant n:\left|d\left(x, A_{k}^{\prime}, B_{k}^{\prime}\right)-L\right| \geqslant \varepsilon\right\}\right|+f(\varepsilon) .
\end{aligned}
$$

Now if $\varepsilon \rightarrow 0$, the theorem is proved. Since $A_{k} \stackrel{Z^{J} W(S)}{\sim} B_{k}$, it follows that the later set belongs to J, and therefore $A_{k} \stackrel{z^{j} W\left(\omega_{f}\right)}{\sim} B_{k}$.

Theorem 3.19. Let $\mathcal{J} \subset 2^{\mathbb{N}}$ be a non-trivial in $\mathbb{N}, \theta=\left\{k_{\mathrm{r}}\right\}$ be a lacunary sequence and $\mathrm{f}$ be a modulus function. If $\liminf f_{r}$ qr $>1$, then $A_{k} \stackrel{Z^{J} W\left(\omega_{f}\right)}{\sim} B_{k} \Longrightarrow A_{k} \stackrel{Z^{J} W\left(N_{\theta}^{f}\right)}{\sim} B_{k}$.

Proof. Assume that $\liminf \operatorname{in}_{\mathrm{r}} \mathrm{qr}>1$, then there exist $\delta>0$ such that $\mathrm{q}_{\mathrm{r}}=\frac{\mathrm{k}_{\mathrm{r}}}{\mathrm{k}_{\mathrm{r}-1}} \geqslant 1+\delta$ for sufficiently large $r$, which implies that $\frac{h_{r}}{k_{r}} \geqslant \frac{\delta}{1+\delta}$. Let $A_{k} \stackrel{z^{j} w\left(\omega_{f}\right)}{\sim} B_{k}$. For sufficiently large $r$, we obtain the following

$$
\begin{aligned}
\frac{1}{k_{r}} \sum_{k=1}^{k_{r}} f\left(\left|d\left(x, A_{k}^{\prime}, B_{k}^{\prime}\right)-L\right|\right) & \geqslant \frac{1}{k_{r}} \sum_{k \in I_{r}} f\left(\left|d\left(x, A_{k}^{\prime}, B_{k}^{\prime}\right)-L\right|\right) \\
& =\left(\frac{h_{r}}{k_{r}}\right) \frac{1}{h_{r}} \sum_{k \in I_{r}} f\left(\left|d\left(x, A_{k}^{\prime}, B_{k}^{\prime}\right)-L\right|\right) \\
& \geqslant\left(\frac{\delta}{1+\delta}\right) \frac{1}{h_{r}} \sum_{k \in I_{r}} f\left(\left|d\left(x, A_{k}^{\prime}, B_{k}^{\prime}\right)-L\right|\right),
\end{aligned}
$$

which gives $\varepsilon>0$,

$$
\left\{r \in \mathbb{N}: \frac{1}{h_{r}} \sum_{k \in I_{r}} f\left(\left|d\left(x, A_{k}^{\prime}, B^{\prime}{ }_{k}\right)-L\right|\right) \geqslant \epsilon\right\} \subseteq\left\{r \in \mathbb{N}: \frac{1}{k_{r}} \sum_{k=1}^{k_{r}} f\left(\left|d\left(x, A_{k}^{\prime}, B^{\prime}{ }_{k}\right)-L\right|\right) \geqslant \frac{\varepsilon . \delta}{1+\delta}\right\} .
$$

Since $A_{k} \stackrel{Z^{J} W\left(\omega_{f}\right)}{\sim} B_{k}$, it follows that the later set belongs to J, and therefore $A_{k} \stackrel{Z^{J} W\left(N_{\theta}^{f}\right)}{\sim} B_{k}$.

Theorem 3.20. Let $(X, \rho)$ be a metric space. Let $\mathcal{J} \subset 2^{\mathbb{N}}$ be a non-trivial in $\mathbb{N}, \theta=\left\{k_{r}\right\}$ be a lacunary sequence, $\left\{A_{k}\right\},\left\{B_{k}\right\}$ be non-empty closed subsets of $X$, and $f$ be a modulus function. Then,

(i) if $A_{k} \stackrel{Z^{j} w\left(N_{\theta}\right)}{\sim} B_{k}$, then $A_{k} \stackrel{Z^{j} w\left(N_{\theta}^{f}\right)}{\sim} B_{k}$;

(ii) $\lim _{t \rightarrow \infty} \frac{f(t)}{t}=\alpha>0$, then $A_{k} \stackrel{z^{J} w\left(N_{\theta}\right)}{\sim} B_{k} \Longleftrightarrow A_{k} \stackrel{z^{J} w\left(N_{\theta}^{f}\right)}{\sim} B_{k}$.

Proof. The proof is similar to the Theorem 3.18 .

Theorem 3.21. Let $(X, \rho)$ be a metric space. Let $\mathcal{J} \subset 2^{\mathbb{N}}$ be a non-trivial in $\mathbb{N}, \theta=\left\{k_{r}\right\}$ be a lacunary sequence, $\left\{A_{k}\right\},\left\{B_{k}\right\}$ be non-empty closed subsets of $\mathrm{X}$, and $\mathrm{f}$ be a modulus function. Then,

(i) if $\mathrm{A}_{\mathrm{k}} \stackrel{Z^{\top} \mathrm{W}\left(\mathrm{N}_{\theta}^{f}\right)}{\sim} \mathrm{B}_{\mathrm{k}}$, then $\mathrm{A}_{\mathrm{k}} \stackrel{Z^{\top} \mathrm{W}\left(\mathrm{S}_{\theta}\right)}{\sim} \mathrm{B}_{\mathrm{k}}$;

(ii) if $\mathrm{f}$ is bounded, then $\mathrm{A}_{\mathrm{k}} \stackrel{\mathcal{Z}^{\mathrm{J}} W\left(\mathrm{~N}_{\theta}^{\mathrm{f}}\right)}{\sim} \mathrm{B}_{\mathrm{k}} \Longleftrightarrow \mathrm{A}_{\mathrm{k}} \stackrel{\mathcal{Z}^{\mathrm{J}} \mathrm{W}\left(\mathrm{S}_{\theta}\right)}{\sim} \mathrm{B}_{\mathrm{k}}$. 
Proof.

(i) Suppose that $A_{k} \stackrel{Z^{\top} W\left(N_{\theta}^{f}\right)}{\sim} B_{k}$ and $\varepsilon>0$ be given. Since

$$
\begin{aligned}
\frac{1}{h_{r}} \sum_{k \in I_{r}} f\left(\left|d\left(x, A_{k}^{\prime}, B_{k}^{\prime}\right)-L\right|\right) & \geqslant \frac{1}{h_{r}} \sum_{k \in I_{r}} f\left(\left|d\left(x, A_{k}^{\prime}, B_{k}^{\prime}\right)-L\right|\right) \\
& \geqslant f(\varepsilon) \frac{1}{h_{r}}\left|\left\{k \in I_{r}:\left|d\left(x, A_{k}^{\prime}, B_{k}^{\prime}\right)-L\right| \geqslant \varepsilon\right\}\right| .
\end{aligned}
$$

If follows that for any $\gamma>0$, if we set

$$
\begin{aligned}
& A(\varepsilon, \gamma)=\left\{r \in \mathbb{N}: \frac{1}{h_{r}}\left|k \in I_{r}:\right| d\left(x, A_{k}^{\prime}, B_{k}^{\prime}\right)-L|\geqslant \varepsilon| \geqslant \gamma\right\}, \\
& B(\varepsilon, \gamma)=\left\{r \in \mathbb{N}: \frac{1}{h_{r}} \sum_{k \in I_{r}} f\left(\left|d\left(x, A_{k}^{\prime}, B_{k}^{\prime}\right)-L\right|\right) \geqslant \gamma f(\varepsilon)\right\},
\end{aligned}
$$

then $A(\varepsilon, \gamma) \subset B(\varepsilon, \gamma)$. Since $A_{k} \stackrel{Z^{\jmath} W\left(N_{\theta}^{f}\right)}{\sim} B_{k}$, so $B(\varepsilon, \gamma) \in \mathcal{J}$. But then, by the definition of an ideal, $A(\varepsilon, \gamma) \in \mathcal{J}$, and therefore, $A_{k} \stackrel{z^{J} W\left(S_{\theta}\right)}{\sim} B_{k}$.

(ii) Suppose that $f$ is bounded and let $A_{k} \underset{z^{J} W\left(S_{\theta}\right)}{\sim} B_{k}$. Since $f$ is bounded there exists a positive real number $M$ such that $|f(x)| \leqslant M$ for all $x \geqslant 0$. Further, using the fact

$$
\begin{aligned}
\frac{1}{h_{r}} \sum_{k \in I_{r}} f\left(\left|d\left(x, A_{k}^{\prime}, B_{k}^{\prime}\right)-L\right|\right)= & \frac{1}{h_{r}}\left[\sum_{\substack{k \in I_{r} \\
\left|d\left(x, A_{k}^{\prime}, B_{k}^{\prime}\right)-L\right| \geqslant \varepsilon}} f\left(\left|d\left(x, A_{k}^{\prime}, B_{k}^{\prime}\right)-L\right|\right)\right. \\
& \left.+\sum_{\substack{k \in I_{r} \\
\left|d\left(x, A_{k}^{\prime}, B_{k}^{\prime}\right)-L\right|<\varepsilon}} f\left(\left|d\left(x, A_{k}^{\prime}, B_{k}^{\prime}\right)-L\right|\right)\right] \\
\leqslant & \frac{M}{h_{r}}\left|\left\{k \in I_{r}:\left|d\left(x, A_{k}^{\prime}, B_{k}^{\prime}\right)-L\right| \geqslant \varepsilon\right\}\right|+f(\varepsilon) .
\end{aligned}
$$

Now if $\varepsilon \rightarrow 0$, the theorem is proved. Since $A_{k} \stackrel{Z^{J} W\left(S_{\theta}\right)}{\sim} B_{k}$, it follows that the later set belongs to J, and hence, the first set in above expression belongs to J. This is proved that $A_{k} \stackrel{z^{J} W\left(N_{\theta}^{f}\right)}{\sim} B_{k}$.

\section{Acknowledgment}

The authors would like to thank the referees and the editor for their careful reading and their valuable comments.

\section{References}

[1] S. Altundag, M. Basarir, On $[\mathrm{W}]_{\sigma, \theta}^{\mathrm{L}}$-Lacunary Asymptotically Equivalent Sequences, Int. J. Math. Anal. (Ruse), 2 (2008), 373-382. 2

[2] A. Esi, On asymptotically double lacunary statistically equivalent sequences, Appl. Math. Lett., 22 (2009), 1781-1785. 1 
[3] A. Esi, M. Acikgoz, On some double lacunary strong zweier convergent sequence spaces, An. Univ. Craiova Ser. Mat. Inform., 40 (2013), 121-127. 1

[4] A. Esi, A. Sapszoglu. On some lacunary 6-strong Zweier convergent sequence spaces, Romai J., 8 (2012), 61-70. 1

[5] J. Fridy, C. Orhan, Lacunary statistical convergence, Pacific J. Math., 160 (1993), 43-51. 2.8

[6] B. Hazarika, A. Esi, On $\lambda$-asymptotically Wijsman generalized statistical convergence of sequences of sets, Tatra Mt. Math. Publ., 56 (2013), 67-77. 1

[7] B. Hazarika, A. Esi, On asymptotically Wijsman lacunary statistical convergence of set sequences in ideal context, Filomat, 31 (2017), 2691-2703. 2.7, 2.10

[8] B. Hazarika, A. Esi, N. L. Braha, On asymptotically Wijsman lacunary $\sigma$-statistical convergence of set sequences, J. Math. Anal., 4 (2013), 33-46. 1

[9] B. Hazarika, V. Kumar, On asymptotically double lacunary statistical equivalent sequences in ideal context, J. Inequal. Appl., 2013 (2013), 15 pages. 1

[10] M. Ilkhan, E. E. Kara, A new type of statistical Cauchy sequence and its relation to bourbaki completeness, Cogent Math. Stat., 5 (2018), 9 pages. 1

[11] M. Ilkhan, E. E. Kara, On statistical convergence in quasi-metric spaces, Demonstr. Math., 52 (2019), 225-236.

[12] E. E. Kara, M. Ilkhan, Lacunary I-convergent and lacunary I-bounded sequence spaces defined by an Orlicz function, Electron. J. Math. Anal. Appl., 4 (2016), 150-159. 1

[13] Y. F. Karababa, A. Esi, On some strong Zweier convergent sequence spaces, Acta Univ. Apulensis Math. Inform., 29 (2012), 9-15. 1

[14] V. A. Khan, K. Ebadullah, A. Esi, N. Khan, M. Shafiq, On paranorm Zweier-convergent sequence spaces, J. Math., 2013 (2013), 6 pages.

[15] V. A. Khan, K. Ebadullah, A. Esi, M. Shafiq, On some Zeweir I-convergent sequence spaces defined by a modulus function, Afr. Mat., 26 (2015), 115-125.

[16] V. A. Khan, A. Esi, Yasmeen, H. Fatima, On paranorm type intuitionisticfuzzy Zweier I-convergent sequence spaces, Ann. Fuzzy Math. Inform., 13 (2017), 135-143. 1

[17] V. A. Khan, H. Fatima, S. A. A. Abdullah, K. M. A. S. Alshlool, On paranorm BV $\sigma_{\sigma}^{\mathrm{I}}$-convergent double sequence spaces defined by an Orlicz function, Analysis (Berlin), 37 (2017), 157-167. 1

[18] V. A. Khan, H. Fatima, S. A. A. Abdullah, K. M. A. S. Alshlool, On Some New I-Convergent Double Sequence Spaces of Invariant Means Defined By Ideal And Modulus Function, Sigma, 35 (2017), 695-706.

[19] V. A. Khan, N. Khan, On Zweier I-convergent double sequence spaces, Filomat, 30 (2016), 3361-3369.

[20] V. A. Khan, R. K. A. Rababah, A. Esi, S. A. A. Abdullah, K. M. A. S. Alshlool, Some new spaces of ideal convergent double sequences by using compact operator, J. Appl. Sci., 17 (2017), 467-474. 1

[21] Ö. Kisi, H. Gümüs, F. Nuray, I-Asymptotically Lacunary Equivalent Set Sequences Defined By A Modulus Function, Acta Univ. Apulensis Math. Inform., 41 (2015), 141-151. 1, 2.5, 2.6, 2.9

[22] P. Kostyrko, T. Šalát, W. Wilczyński, I-convergence, Real Anal. Exchange, 26 (2000/01), 669-685. 1, $2.3,2.4$

[23] I. J. Maddox, Sequence spaces defined by a modulus, Math. Proc. Cambridge Philos. Soc., 100 (1986), 161-166. 2

[24] M. S. Marouf, Asymptotic equivalence and summability, Internat. J. Math. Math. Sci., 16 (1993), 755-762. 1

[25] H. Nakano, Concave modulars, J. Math. Soc. Japan, 5 (1953), 29-49. 2

[26] R. F. Patterson, On asymptotically statistically equivalent sequences, Demonstratio Math., 36 (2003), 149-153. 1

[27] F. Patterson, E. Savas, On asymptotically lacunary statistical equivalent sequences, Thai J. Math., 4 (2012), 267-272. 1

[28] I. P. Pobyvanets, Asymptotic equivalence of some linear transformation defined by a nonnegative matrix and reduced to generalized equivalences in the sense of cesàro and abel, Mat. Fiz., 28 (1980), 83-87. 1

[29] M. Sengonul, On the Zweier sequence space, Demonstratio Math., 40 (2007), 181-196. 1

[30] N. Subramanian, A. Esi, Wijsman rough $\lambda$-weak statistical convergence of order $\alpha$ of triple sequence of functions, Asia Pacific J. Math., 4 (2017), 49-61. 1

[31] N. Subramanian, A. Esi, On asymptotically f-rough statistical equivalent of triple sequences, J. Appl. Math. Inform., 37 (2019), 459-467.

[32] U. Ulusu, E. Dündar, Asymptotically I-cesáro equivalence of sequences of sets, Univ. J. Math. Appl., 1 (2018), 101-105. 1

[33] U. Ulusu, F. Nuray, Lacunary statistical convergence of sequences of sets, Progress Appl. Math., 4 (2012), 99-109. 1

[34] U. Ulusu, F. Nuray, On asymptotically lacunary statistical equivalent set sequences, J. Math., 2013 (2013), 5 pages. 2.1, 2.2 